\title{
Instability strip for pre-main-sequence stars
}

\author{
A. Grigahcène ${ }^{1,2}$, M.-A. Dupret ${ }^{3}$, R. Garrido ${ }^{2}$, M. Gabriel ${ }^{4}$ \\ and R. Scuflaire ${ }^{4}$ \\ ${ }^{1}$ Algiers Observatory, CRAAG, BP 63 Bouzaréah, 16340, Algiers, Algeria \\ ${ }^{2}$ Instituto de Astrofísica de Andalucía-CSIC, Apartado 3004, 18080 Granada, Spain \\ ${ }^{3}$ Observatoire de Paris, LESIA, 92195 Meudon, France \\ ${ }^{4}$ Institut d'Astrophysique et de Géophysique de I'Université de Liège, Belgium
}

\begin{abstract}
We apply the Time Dependent Convection (TDC) treatment presented in Grigahcène et al. (2005) to the study of Pre-main sequence pulsating stars. The stabilization of the p-modes at the red edge of the Instability Strip (IS) is obtained by our models. The theoretical IS obtained with Mixing Length (ML) parameter $\alpha=1.8$ is compared to observations and a good agreement is obtained.
\end{abstract}

\section{Introduction}

There is a growing interest in studying the first stages in the formation of stars, and a need to know more on the detailed structure and evolution of single stars from the protostellar phase to the main sequence, and the nature of the processes that determine and regulate the onset of star-formation within molecular clouds in the first place (Marques et al. 2004). Pre-main sequence (PMS) stars are very active stars showing winds, jets, outflows etc. They are interacting with the circumstellar environment in which they are embedded.

They show photometric and spectroscopic variability on time scales from minutes to years. On time scales of half an hour to some hours the variation may be due to pulsation if the star lies in or near the instability strip. Intermediate mass stars can become pulsationally unstable during various stages of their evolution. The fact that young stars during their evolution to the main sequence move across the instability region raises the possibility that at least part of the observed stellar activity could also be due to stellar pulsations (Kurtz, 2000; Marconi \& Palla 1998). The use of pulsations in PMS stars may provide a unique means for constraining the internal structure of PMS stars and for testing evolutionary models.

Recently, the use of Time-Dependent Convection (TDC) permitted us the determinations of theoretical IS for $\delta$ Sct and $\gamma$ Dor (Dupret et al. 2005). In this work, we apply this treatment to PMS models to investigate the pulsational properties of PMS stars.

\section{Mode stabilization}

In this section, we show that the stabilization of radial modes at the red edge of the IS is obtained with our TDC treatment. In Fig. 1, we give the evolution (from PMS stage to TAMS) of the frequencies $\sigma(\mathrm{c} / \mathrm{d})$ of $\ell=0$ modes as a function of the age for $1.8 M_{\odot}$ models with a ML parameter $\alpha=1.8$ (solar calibrated value for our evolutionary code). We note two clear regions during the evolution, the first corresponding to the IS of the PMS stage, the 


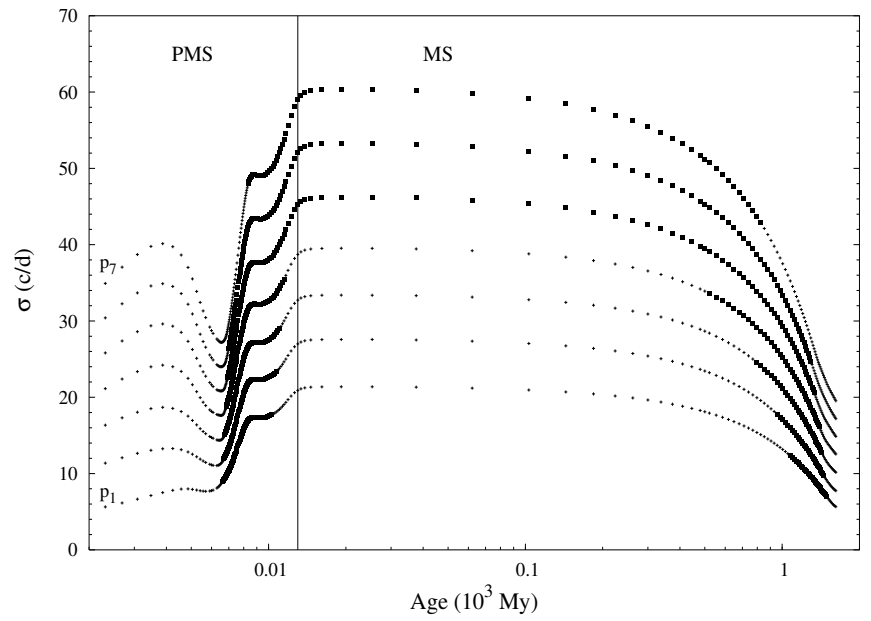

Figure 1: Evolution of frequencies of the stable (plus symbol) and unstable (black square symbol) modes for stellar models of $1.8 \mathrm{M}_{\odot}$ with $\alpha=1.8$. The vertical line shows the ZAMS point. In its left we have the PMS models while in its right we have MS models.

second to the MS stage. These two instable regions are not separated for the modes $\mathrm{p}_{5}, \mathrm{p}_{6}$ and $\mathrm{p}_{7}$ because the ZAMS point is inside the IS for a $1.8 M_{\odot}$ evolutionary track. The figure shows that our TDC models succeed in stabilizing the radial modes at low temperatures (we chose to represent the evolution in function of age and not effective temperature for reasons of clarity), leading therefore to both IS red edges. The frequency range of the unstable modes predicted by our models is in agreement with the typical observed frequencies of $\delta$ Sct stars (Suran et al. 2001).

\section{PMS theoretical Instability Strip}

In order to determine theoretical IS, a large number of evolutionary tracks have been computed, with masses ranging from 1.4 to $2.2 M_{\odot}$. Performing non-adiabatic pulsation computations for all these stellar models, we have determined the theoretical IS for PMS stars obtained with our TDC treatment. In Fig. 2, we present the theoretical IS obtained for radial modes, for models with $\alpha=1.8$. Each curve corresponds to the blue or red edge of a mode of given radial order $n$. Labels enable to identify the modes (e.g. $\mathrm{p}_{1 \mathrm{R}}$ for the red edge of the $\mathrm{p}_{1}$ mode). As the radial order of the modes increases, the blue and red edges are shifted towards higher $T_{\text {eff }}$. The small points correspond to the position of the 25 observed pulsating PMS stars of the AMS working group catalogue.

For comparison, we also give in Fig. 2 the location of the theoretical IS boundaries of the fundamental, first and second overtones radial modes obtained by Marconi \& Palla 1998 (broken dotted lines). We can see that half of the observed pulsating PMS stars could oscillate in the fundamental radial mode, while the rest could oscillate in the high order modes. Some stars stay out of the IS, they could be chemically peculiars. Hence, more observations are needed to know more about them. 


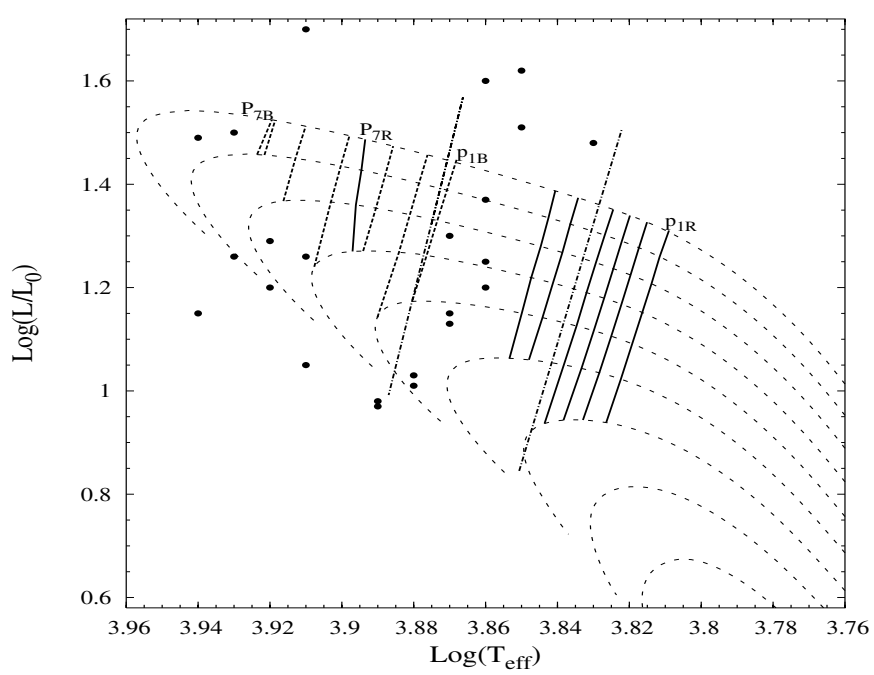

Figure 2: Blue and red edges of the PMS theoretical IS for radial modes from $\mathrm{p}_{1}$ to $\mathrm{p}_{7}$ for models with $\alpha=1.8$. Small points correspond to observations. Broken dotted lines give the IS of Marconi \& Palla (1998).

\section{Conclusions}

Following the investigations done on the pulsation properties of intermediate mass stars (see Dupret et al. 2005), we considered in this work the first stages in the evolution of these stars. We used our TDC treatment presented in Grigahcène et al. (2005) and we applied it to the study of the driving and damping mechanisms in PMS stars. We succeed in obtaining both the blue and red edges, for radial modes. We get a good agreement with observations for models with $\alpha=1.8$ (solar calibrated value). Our future prospect is to consider the non-radial modes, to compare PMS and MS stars using photometric observables.

\section{References}

Dupret, M.-A., Grigahcène, A., Garrido, R., Gabriel, M., Scuflaire, R. 2005, A\&A 435, 927

Grigahcène, A., Dupret, M.-A., Gabriel, M., et al. 2005, A\&A 434, 1055

Kurtz, D. W. 2000, in "Delta Scuti and Related Stars", eds. M. Breger, M.H. Montgomery, ASP Conf. Ser. 210, 287

Marconi, M., Palla, F. 1998, A\&A 507, L141

Marques, J. P., Fernandes, J., Monteiro, M. J. P. F. G. 2004, A\&A 422, 239

Suran, M., Goupil, M., Baglin, A., et al. 2001, A\&A 372, 233 